\title{
UNDERSTANDING THE PHYSICAL WORLD
}

\author{
By William V. Houston
}

THE ASSOCIATION OF RICE ALUMNI has done me a

1 great honor in inviting me to inaugurate this series of lectures. I appreciate it. I hope this activity on the part of the Alumni will lead to spreading understanding of what a university is for, of why we believe centers of research and advanced teaching are a vital part of our civilization.

This is the fiftieth year in the active life of Rice University. At the time of the opening in the year 1912 many things in our later development were foreshadowed. I have been particularly impressed by two. In the first place, I find very interesting the series of statements made by President Edgar Odell Lovett about the new university. He was specific in stating that there would be no upper limit placed upon the work at Rice; and that because of its importance in the coming twentieth century, the new institution would first be developed in the direction of the physical sciences.

And then the reality of the principles announced by the president was symbolized by a letter dated October 25, 1912, and published in the Philosophical Magazine of London and Edinburgh for that year. It was dated from the Rice Institute just a few days after the opening ceremonies and was written by Professor H. A. Wilson. He was already at work and reporting his results from this laboratory.

EditoR's Note: This lecture was delivered in Hamman Hall on February 21, 1962, as the first of the Rice Alumni Association's Distinguished Scholar Program. Dr. Houston was President of Rice University from 1946 to 1960 . He is presently Honorary Chancellor of the University and Distinguished Professor of Physics. He is President of the American Physical Society for 1962-1963. 
Because of its association with the first fifty years of this University, it seemed to me appropriate to recall this evening one of the ways in which twentieth-century physics has forced a revision in some of our cherished modes of thinking. I do not propose to talk about gadgets or machines, large or small. Such machines are essential to the exploration and investigation of the physical world. But I hope to emphasize some of the basic facts discovered with these machines, and, in particular, to emphasize the contention that we learn from these facts certain epistemological lessons which can perhaps be carried into other fields. Epistemology is a long word with some technical philosophical connotations. I like to think of epistemology as the effort to find out if one knows what he is talking about. And so an epistemological lesson may be paraphrased as a lesson as to the extent one does or does not know what he is talking about.

It is sometimes said that the modern way of looking at the natural world began with Galileo. Historically, this is an oversimplification, but Galileo can well be used to symbolize the rise of experimental science. We now take it for granted that to learn about anything we must examine it, watch it, interfere with it, experiment on it. When we do this, we try to describe our observations and conclusions. This process may seem simple and straightforward, but twentieth-century developments in physics have shown that there can be many a pitfall in the words we use, and in the descriptions we give.

Some years after Galileo, as you know, Newton formulated the laws of mechanics and his law of gravitation. In doing so, he was led to talk about particles of matter, or corpuscles. He was not referring to anything like fundamental or indivisible atoms. He was referring merely to pieces of matter so small he felt he had no need to consider their size and extension in space.

As a consequence of Newton's work and later develop- 
ments, the conviction grew that all material behavior could be described in terms of particles pushing at each other, or moving freely under the influence of forces from more distant objects, such as forces of gravitation, electrostatic attractions and repulsions, or magnetic forces. Presumably, if a particle was small enough and far enough away from all others, it could be expected to move in a straight line with uniform velocity. When it didn't move uniformly in a straight line, the departure was regarded as evidence of a force whose nature could be inferred. And so one had a picture of a material world, composed of many pieces, moving in orderly ranks under the influence of forces, some of which were known, and some of which were still to be identified.

When the existence of actual chemical atoms was demonstrated during the first part of the nineteenth century, most people, with a few notable exceptions, began to picture the actual physical existence of atoms as small particles not further divisible, but whose motions could be described by means of the same strict mechanical laws formulated by Newton. Developments in techniques toward the end of the century led people to undertake experiments to justify such views. It proved not too difficult to show the existence of the atoms and, later, of their constituent particles. It was more difficult to be sure their motions could be described mechanically. Nevertheless, the hope persisted that a detailed mechanical explanation of all observed phenomena would eventually be possible.

But not all physical phenomena are easily described by devoting attention to individual particles. In large bodies of liquids or solids there are many particles close together, acting on each other directly. It is often convenient to describe internal motions of these bodies, not by describing the motion of the individual particles, but by describing a general motion of the body in the form of a wave.

The surface wave on a body of water gives simple visual evidence of wave motion. If one touches the surface of a 
pool of still water, or drops a small pebble into it, ripples go out from the center in circles of increasing radius. Figure 1 shows a number of concentric circles intended to remind us of this kind of behavior, which we have all seen. Presumably, these circles continue traveling indefinitely on the surface of the water unless they meet an obstacle. But in and around obstacles, there are some very peculiar happenings, peculiar, that is, to wave motion. If the waves meet a solid obstacle, they are reflected in various ways. If they meet an

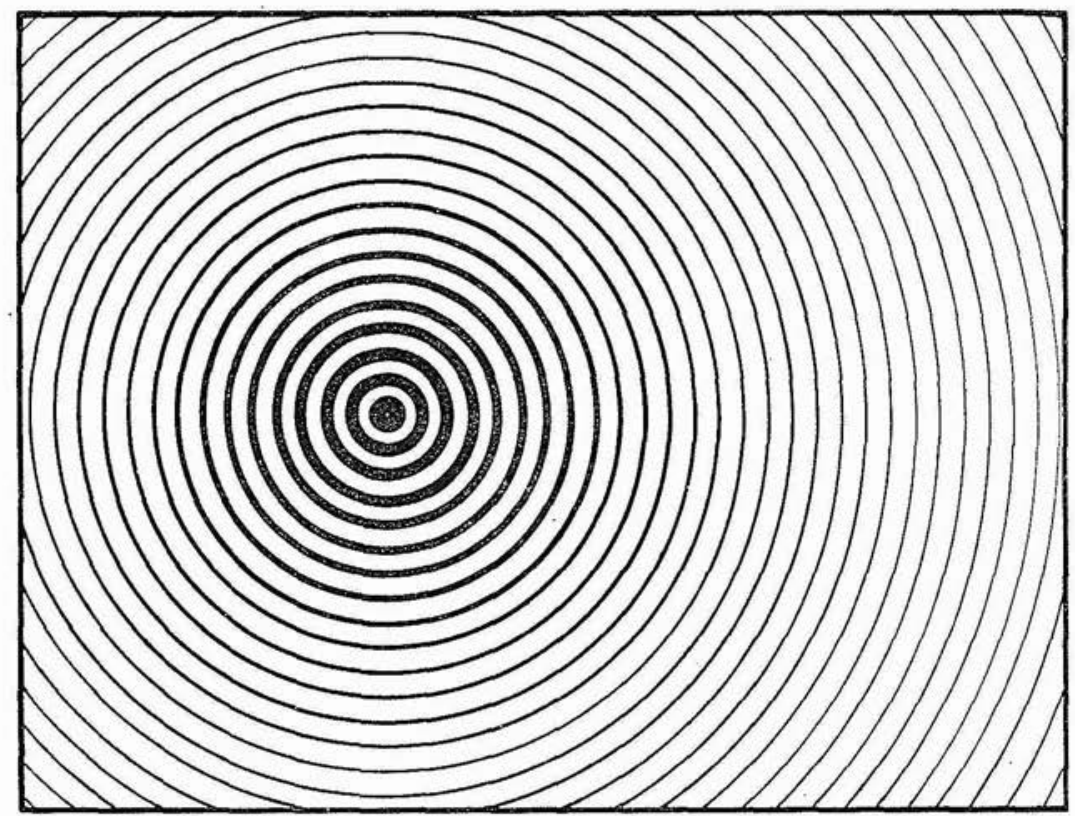

FIGURE 1.-A wave started on the surface of a liquid spreads out in all directions.

obstacle with a small opening in it, some of the wave passes through the opening and on the other side tends to spread out as though radiating from a new source. Figure 2 is designed to suggest this situation. The large circles to the extreme left represent waves from a distant source that meet a solid barrier with a hole in it. Some of the wave goes through, and we concentrate attention on it, ignoring that 


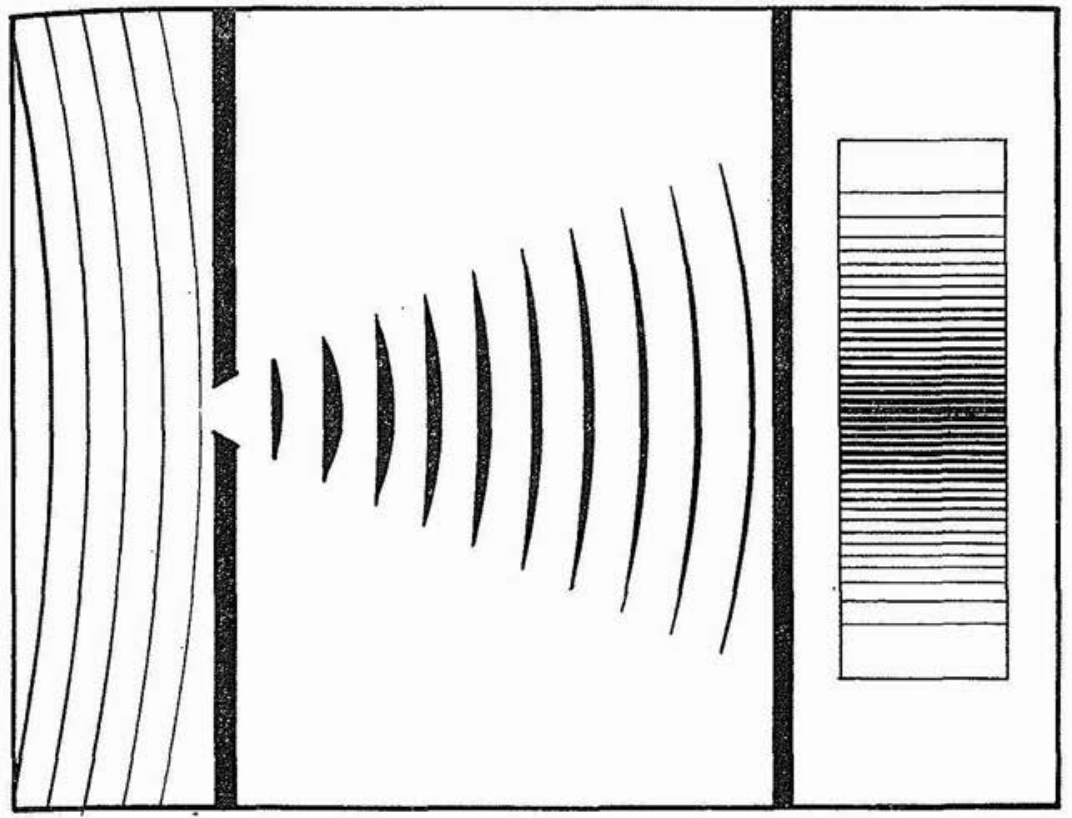

FIGURE 2.- When a wave meets an obstacle with a small opening, part of the wave goes through the opening and spreads out as from a new source. If the size of the opening is about the wave length of the wave, there is no sharp shadow on a screen. There is a pattern with maximum intensity in the center and smoothly decreasing intensity on each side. The panel at the right of the figure gives the intensity as viewed by an observer from the right.

part of the wave which is reflected. If then one examines this wave at some distance beyond the barrier, we find it is an intense wave at those places directly in line with the opening and the intensity falls off on both sides. The marks to the right side of the figure are intended to illustrate this situation. When the width of the opening in the obstacle is not too much greater than the wave length of the wave, there is no sharp shadow, but what is called a diffraction pattern. It fades off gradually on both sides.

Still more strikingly, if there are two apertures through which the wave can go, the maximum intensity appears at a point not opposite to either opening but halfway between them, as shown in Figure 3. This is a somewhat more complicated diffraction pattern than is produced by a single 


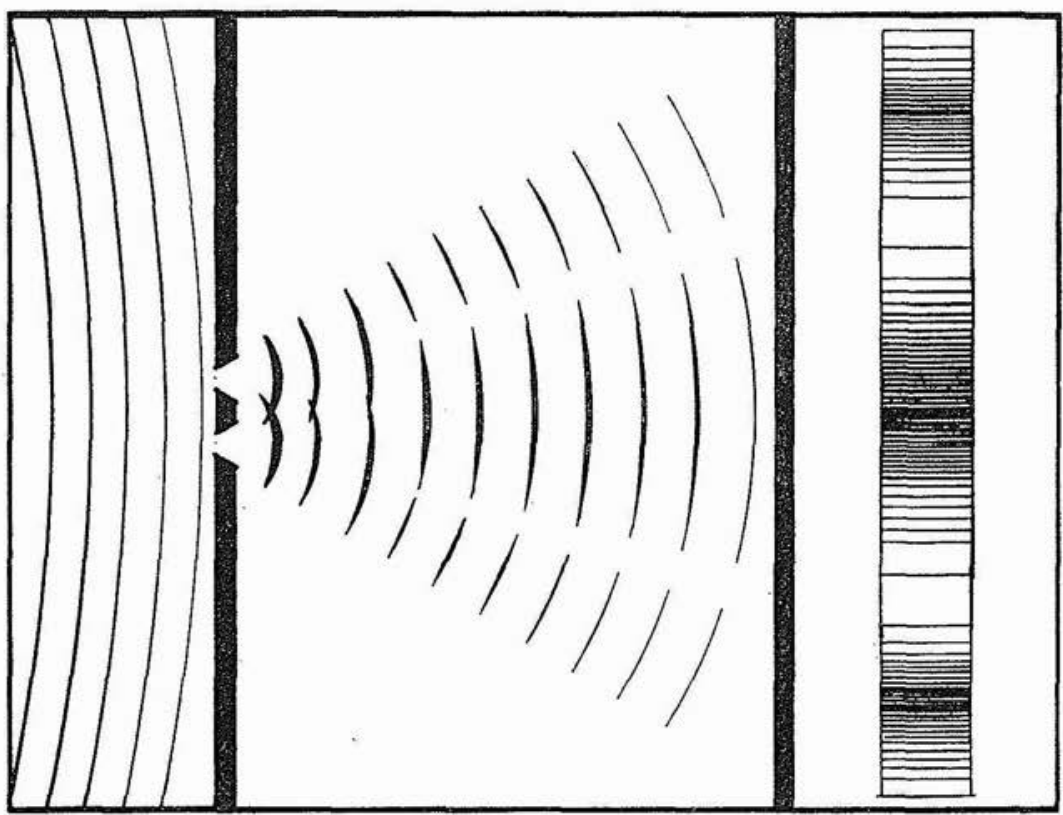

FIGURE 3.-When a wave meets a barrier with two openings of suitable size and separation the wave goes through them to form a characteristic pattern on a screen. The maximum of intensity is at the center, equidistant from the two openings. The maximum has four times the intensity produced when only one opening is present, and there are positions of lower intensity than when only one opening is present. The intensity pattern is not at all a superposition of the patterns due to the individual openings.

aperture. The important thing, however, is that the pattern of intensity shown on the right is not at all a superposition of the individual patterns of the two separate openings. Waves from these two openings interfere in some directions and produce a wave intensity less than would be present if there were only one aperture. By various arrangements of slits and holes and obstacles, quite complicated diffraction patterns can be produced. The nature of the diffraction pattern due to such situations can be readily calculated and predicted.

These diffractions and interference patterns can be demonstrated on the surface of a liquid with appropriate equipment. They can also be demonstrated in the case of sound waves in air or in water. There are sometimes particular 
places in an auditorium at which the sound from the speaker's desk is weak or even inaudible. Such demonstrations provide conclusive evidence that sound is carried by a wave motion in a gas or liquid, such as air or water, or by a similar elastic wave in a solid.

And so there were developed two very distinct limiting cases of mechanical ideas. On the one hand was the idea of corpuscles, or small particles of matter, or atoms, tending to move in straight lines and generally obeying Newton's law of motion. On the other hand there was the idea of a mechanical wave in an all-pervading medium such as air or water. The medium itself could be thought of as composed of particles pushing on each other, but the wave motion was a collective motion of the system as a whole.

There are events in the physical world, however, which are not mechanical. There is light for example. But one tries to understand light in terms of simple mechanical ideas. The

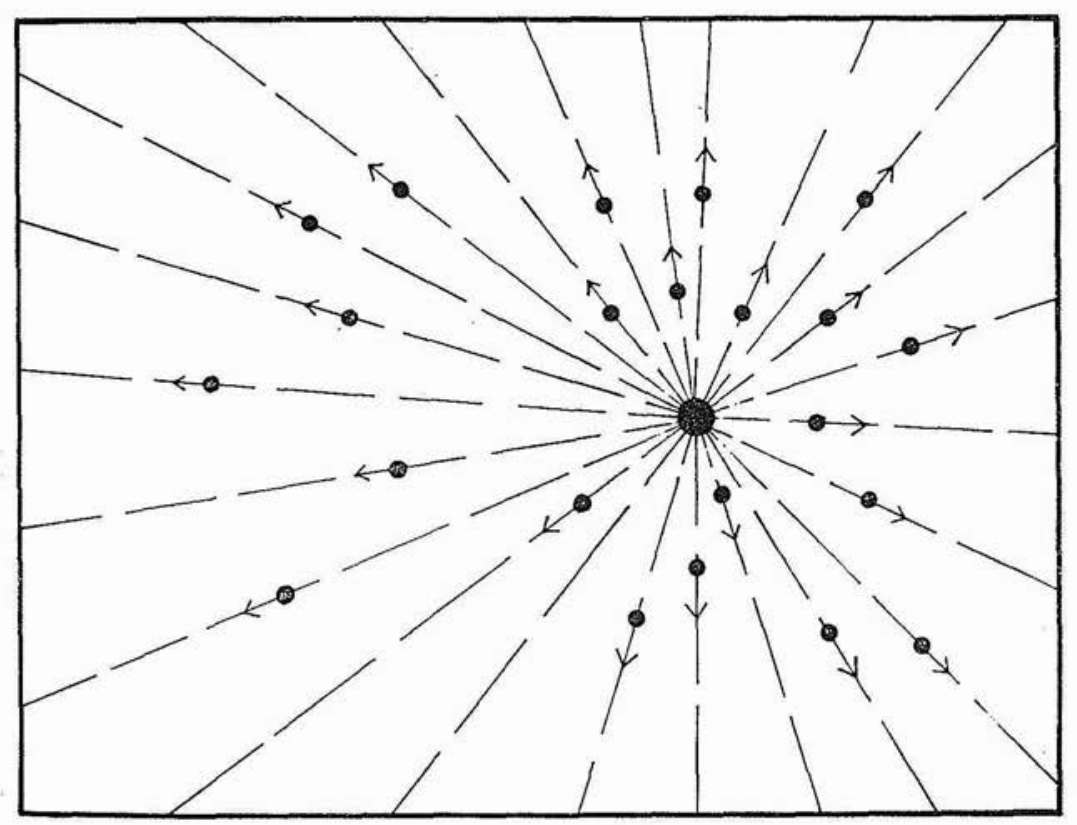

FIGURE 4.-Particles emitted from a small source will travel in straight lines in all directions. 
idea of light as a stream of particles is very old, and satisfactorily describes the phenomena of geometrical optics. Figure 4 shows what might be thought of as a series of light particles shot out in straight lines from the source. Such particles would be reflected by obstacles and would change direction under the influence of suitable forces. This figure can be contrasted with Figure 1 which shows a series of circles. If such a system of particles were to strike an obstacle containing an opening, Figure 5 shows what is to be expected. Some

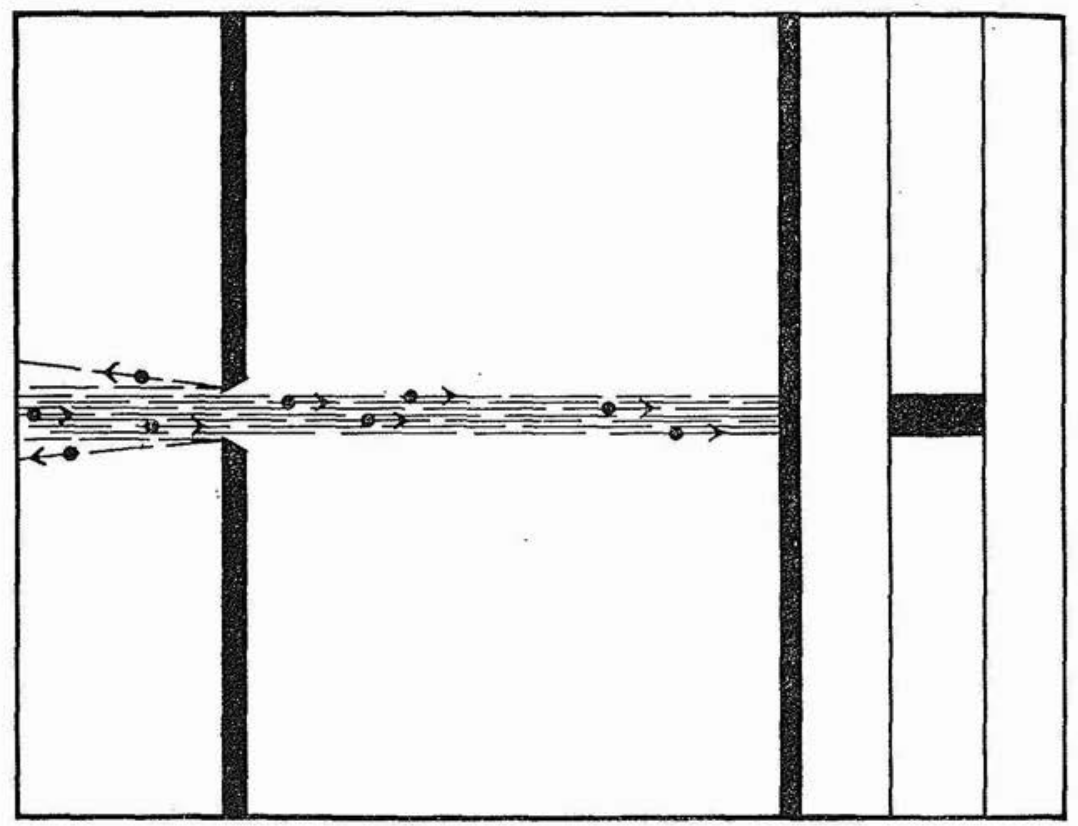

FIGURE 5.-When particles from a distant source meet a barrier containing an opening, they will be reflected or will pass through in straight lines to form a sharp shadow on a screen.

particles pass through the opening and some do not. If they hit the opening, they will go straight through and hit the screen on the right-hand side of the figure in such a way as to form a sharp shadow of the opening. This is what happens in many cases, and so this picture of light was satisfactory for many purposes.

However, during the first decades of the nineteenth cen- 
tury, many examples of interference and diffraction were established for light. All of the phenomena associated with water waves and elastic waves seemed to be present in the case of light and with such a wave picture a calculation of the distribution of light intensities for all sorts of experimental arrangements could be accurately made. The evidence seemed overwhelming that light must be described as a wave motion. During the whole of the nineteenth century hardly anyone had the temerity to question this conclusion and the straightforward interpretation of the observations. Light must be a wave motion and the apparent sharp shadows cast by objects were only evidence of the shortness of the wave length of the light waves.

Occasionally someone would raise the embarrassing question as to the kind of medium in which these light waves traveled. Some minds were satisfied by giving it a name. It was called the "luminiferous ether." Some people then tried to find properties of this ether. It was necessary that every object, such as a baseball, for example, or even the earth itself, be able to move through it without meeting any resistance. On the other hand, it was necessary for it to show such rigidity that only transverse waves could propagate. Well I remember Professor A. A. Michelson, the first American winner of a Nobel prize in physics, undertaking to make plausible this kind of description of the ether. He pointed out that a stick of sealing wax at room temperature is brittle and will break if subjected to a sharp blow. On the other hand, if the sealing wax is subjected to a small but continuous force for a long period of time it will bend and not break. The implication was that fluid flow and high rigidity need not be mutually exclusive if widely differing velocities are under consideration. But even this picture of the ether was seriously discredited by the Michelson-Morely experiment, which was the first demonstration that the velocity of light is a velocity relative to no ether of any kind, but relative only to the observing instruments. After 1900, the term ether 
began to fall into disrepute, and light was regarded as wave motion in the electromagnetic field. Somehow the words "electromagnetic field" seem to alleviate some of the difficulties suggested by the word ether.

In spite of this somewhat esoteric discussion as to the nature of the wave-carrying medium, there could be no question that the results of all experiments involving interference or diffraction of light could be described only by reference to some kind of wave motion. The facts of geometrical optics were then referred to the extreme shortness of the waves, and so all seemed to be in order. This situation was emphasized further by the demonstration about 1912 of the wave nature of $\mathrm{X}$ rays and the beginning of a long series of experiments which successfully measured such very short wave lengths.

Thus at the end of the century, the probings of physicists and chemists into the structure of matter and the nature of electromagnetic phenomena seemed to have revealed two kinds of ultimate pictures. On the one hand, there were electrons and atomic nuclei, expected to obey the laws of mechanics; and on the other hand, there were wave phenomena which could be described by Maxwell's equations of the electromagnetic field. The nature of the interaction between these two was not clearly understood. Presumably, moving electrons could produce electromagnetic waves in the same way a stone dropped into water produces a water wave. The details of this process had not been worked out, but it was confidently expected that they would be understood in due time. There was where the trouble began.

One of the first things observed in the early years of the twentieth century was that light does not behave as a wave motion in all respects. When a diffraction pattern is photographed with a long enough exposure, the distribution of density on the photographic plate is that to be expected from the intensity of a wave. If, however, the photographic plate is replaced by a fluorescent screen, and the intensity of the 
light, or the $\mathrm{X}$ rays, or the gamma rays, is made sufficiently low, light particles can be seen striking the screen one at a time. After a long enough period these individual flashes will fill up the diffraction pattern, but it is clear that they have arrived one at a time.

Figure 6 is an attempt to illustrate what $I$ have been saying. The strip " $\mathrm{A}$ " at the top indicates what is to be expected

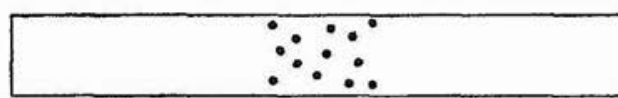

A

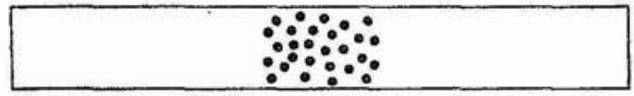

B
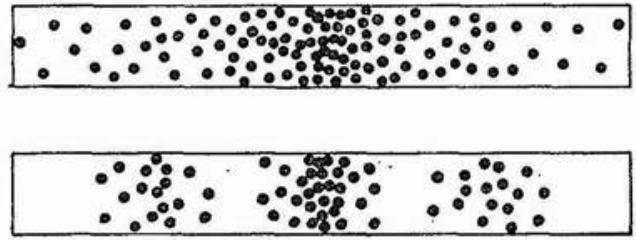

D

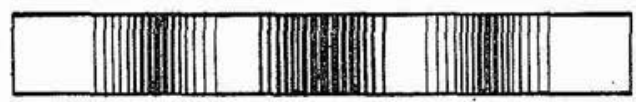

$E$

FIGURE 6.-A. Particles passing through an opening strike a screen one at a time but always within the limits set by the geometry of the screen and the opening.

B. Longer "exposure" leads to a more dense covering of the screen by individual impacts, but they all lie within limits set by the geometry.

C. When light or X rays pass through a small opening, individual impacts are recorded on the screen, but they are not restricted to the limits expected for particles. The impacts are distributed with a density corresponding to the intensity expected for a wave.

D. If light passes through two openings, the individual impacts form an interference pattern.

E. A long enough exposure brings enough individual impacts to form an interference pattern and conceal the individual spots.

if a small number of particles comes through a slit and strikes the screen. Each impact makes a spot within the limits permitted by the slits through which they have passed. After 
a longer time more particles have come through and make a denser pattern such as shown in " $B$ ", but still the pattern is sharp. If, however, light or $\mathrm{X}$ rays pass through a slit, narrow compared to their wave length, they make spots on the screen, as in " $C$ ", but these spots are not confined to an image of the slit but spread out on both sides. If instead of one slit the light or the $\mathrm{X}$ rays pass through two slits side by side, a pattern such as in " $\mathrm{D}$ " is produced, and the striking thing is that there are some places in " $D$ " with fewer spots than in "C". Then, of course, if the light continues coming through, the spots pile up more and more densely to give the well-defined pattern at " $E$ ".

This complex of observations certainly throws grave doubt on a simple wave picture.

A wave is a large affair. It occupies a significant part of the medium in which it moves. It seems inconceivable that a phenomenon properly described as a wave motion could lead to a succession of individual impacts on a screen. One might be inclined to return to the idea that light must consist of particles traveling from the source to the detecting screens along paths described by the laws of mechanics and suitably formulated forces. This description, however, seems excluded by the nature of interference or diffraction patterns. In particular, if the light is permitted to pass through two or more openings before striking the screen, the apparent particles will strike in certain places. They will not, however, strike the screen in some of the places where they would have struck, had only one opening been available to them.

The experimental observations clearly present a dilemma. Is light to be described as a wave motion or as a stream of particles? In some kinds of experiments the wave nature of light seems unmistakable. In other kinds of experiments its particle nature seems just as clear and convincing. One may ask over and over, what is really the nature of light? Eventually one becomes more sophisticated and inquires only, 
what can be said about light, how will light behave under different conditions? It is in recognizing such limitations on our means of description that the physicists of this century have made a significant contribution to philosophical thought.

During the first quarter of the century, this dual nature of electromagnetic radiation became grudgingly accepted. Then about 1927 our well-established modes of thinking were again rocked when it was announced by Davisson and Germer in this country and by George Thompson in England that electrons can be diffracted and can interfere with themselves. I say interfere with themselves because it is not that one electron interferes with another, but it seems to interfere with itself. If, for example, a beam of electrons is permitted to pass through two openings and to form on the screen an interference pattern, it cannot be said that some electrons go through one opening and some through the other. It can only be said that each electron goes through both slits. We can only say that each electron acts like a train of waves, at least until its presence is made known on a fluorescent screen. The evidence is just as good, in fact it is the same, for electrons as for light and $\mathrm{X}$ rays.

Thus we have a real dilemma. Instead of a nice separation of the material world into waves and particles, we seem to have waves and particles all mixed up together. Only our thinking is separated into waves and particles.

In general, there have been two ways in which philosophically minded physicists and physically sophisticated philosophers have received this disturbing evidence of their eyes. The first reaction is simply an extreme reluctance to consider abandoning our conventional modes of thought, even in the face of such obvious difficulties. Representatives of this view have been unwilling to regard the kind of experiments I have described as ultimate experiments. They prefer to believe that further study and investigation will reveal additional details, sufficient to restore a description 
of electrons and of light to a basis more natural and pictorial in nature.

Possibly the most widely recognized exponent of this school of thought was Albert Einstein, who, as you know, devoted a good deal of his last years to the effort to push beyond the apparent paradoxes and to imagine some way in which the motion of an electron could be described in terms of a well-defined trajectory, some way in which a detailed determinism could be restored to the physics of light and electrons. Another distinguished physicist who has been reluctant to regard the experimental situation as more than temporary is Louis de Broglie. Although he was the first to suggest application of wave ideas to the motion of electrons in atoms, he has found it difficult to believe that the contradictions will not eventually be resolved in terms of our more familiar concepts. Recently, he has written again in amplification of his efforts to combine the wave and particle idea so as in some way to regard the waves as guiding the particles. A few years ago, David Bohm also undertook to find a way out of a purely statistical interpretation of the experimental results. He invoked a kind of "quantum mechanical force" to describe the apparently erratic behavior of electrons when they form a diffraction pattern. So far as I can tell, these efforts have not been successful, and, although many persons are unhappy concerning the apparent necessity for abandoning detailed causality in atomic physics, no satisfactory method of retaining it has yet been proposed.

The other, and I believe more profitable reaction, is represented most eloquently and profoundly by the position of Niels Bohr. According to Dr. Bohr, we must abandon our conventional ways of speaking. We must abandon our intuitive desire to say that an electron is a particle or is a wave. We must content ourselves with a mathematical formulation which permits us to describe, and in fact to predict, the result of any experiment. In so doing, we must take account of all of the apparatus involved in the experiment. We must 
treat the experiment as a whole. We must describe the experiment rather than the object experimented upon. We cannot clearly separate the object from the means of studying it. Dr. Bohr has given the name "complementarity" to this point of view. The wave description and the particle description complement or complete each other. Complementarity recognizes the inadequacy of our simple concepts and our simple language for describing the nature of fundamental objects, and yet recognizes also that only in terms of such concepts and in such language can the nature and the results of experiments be formulated and described. In discoursing about these matters at the Rice University recently, Dr. Bohr expressed some concern because relatively few physicists and almost no professional philosophers have given extensive careful attention to these problems which he believes so important.

It seems to me that we have to recognize here two points. In the first place, there is a long series of experiments, similar to those I have described, whose results cannot all be interpreted in terms of a simple consistent picture of electrons and light. And yet, pictorial descriptions, or descriptions in common language, are the only kind possible, and these must be made from at least two apparently contradictory and mutually exclusive points of view. These points of view are quite properly described as complementary.

In the second place, however, it has been possible to build up a mathematical formalism by which the results of many experiments can be described and predicted. In some cases, these results and predictions are statistical in nature; and this fact represents an abandonment of what one might expect from a satisfactory theory. For a working physicist the situation is not too unsatisfactory. It is only when one wishes to describe the experiments in terms of a picture or a model that many people become unhappy. The efforts of Einstein, de Broglie, and Bohm have been to interpret or extend the mathematical formalism so that a satisfactory pictorial in- 
terpretation may be possible. They do not seem to have succeeded.

Dr. Niels Bohr, on the other hand, has emphasized and reiterated his belief that no such interpretation is possible. $\mathrm{He}$ bases his conviction on the very nature of the ideas involved in describing particles and their motion. He points out also that any experiment must be treated as a whole. One cannot subdivide an experiment indefinitely any more than one can subdivide a piece of matter indefinitely. This fact can be illustrated with reference to the interference of an electron with itself when it passes through two slits before impinging on a detecting screen. If one tries to analyze this experiment further-for example, by trying to determine through which slit the electron passes-it can be done only by blocking one slit. This act changes the experiment entirely. It changes the interference pattern in which the electrons may be expected to distribute themselves. It is a different experiment, and no information concerning the path of an electron in the first experiment can be obtained in such a way.

It is Dr. Bohr's contention that the very ideas used are entirely inadequate to describe an electron, but must be used to describe an experiment with electrons, for no other language is available.

It is furthermore Professor Bohr's contention, and a contention he likes to emphasize, that this abandonment of the possibility of describing an electron in familiar terms does not involve an abandonment of our desire to understand the physical world in greater detail. In fact, Bohr would say it is only when we abandon the desire to describe an electron as either a particle or a wave that we bring ourselves into a position in which we can actually look at the experiments as they are. We must look at the world as it is, and then formulate a precise and satisfactory theory, such as quantum mechanics seems to be, of the behavior of the electrons under all circumstances. 
This point of view seems very much an extension of the point of view required by Einstein in his interpretation of the Michelson-Morely experiment. According to Einstein, the experiment must be accepted at face value. It must be concluded that the velocity of light is constant, relative to any and all observers. Such a conclusion seems, at first, entirely irrational. In view of our ordinary methods of thinking, it is self-contradictory. But the great genius of Einstein lay in the fact that he was willing to accept the experimental observation at its face value, was able to formulate the conclusions that follow from it in a precise way, and was able to develop his theory of relativity. Such a point of view is merely the point of view which, we say, started with Galileo. We say that one must look at the physical world and accept it as it is. We must not undertake to describe it by introspection, or by intuition.

And the next step in this process is that advocated by Dr. Niels Bohr. It goes further than the generalization of Einstein, but, in this sense, it goes in the same direction. By accepting at face value the results of experiments, mutually contradictory as they may seem, and by formulating their logical and mathematical consequences, we can proceed to a more and more profound understanding of the physical world. This, it seems to me, is the great forward step of physics thus far in the twentieth century.

Dr. Bohr has also pointed out that this general idea, this epistemological lesson as he calls it, may well have applications far beyond the field of physics. He suggests some. For example-in the field of biology there have been from time to time at least two apparently contradictory philosophies. There has been the philosophy according to which biologists undertake to explain the characteristic features of living organisms in terms of mechanical laws applied to their smallest parts-and these smallest parts must be at least as small as electrons and atomic nuclei. On the other hand, there has been a philosophy that might be called vitalism. 
According to this view, there is more to a living organism than the mechanical operation of its smallest parts. Something else is required to explain the evolving nature of life.

Most working biologists are guided, when they think of it, by the mechanistic view. They are engaged in the analysis of biological processes and the attempt to push that analysis as far as possible. Many of them recognize, however, a situation somewhat similar to that observed in physics. If one carries out experiments on a living cell in such a way as to determine the mechanical behavior of its atoms, the cell is killed. The cell can exist as living organism only as long as experimental approaches to it do not become such as to define sharply its components.

My understanding of Dr. Bohr's position is that he would say these two aspects of living matter, which might seem to be mutually exclusive, are in fact complementary. He would say that it may be possible, by abandoning one's desire to adhere exclusively to one or the other of these apparently contradictory views, to develop a description of living matter based on the facts of observation and more useful and more inclusive of all types of experiments than has been hitherto developed.

A short time ago Professor Wann of Rice told me that there seems to be developing what might be thought of as analogous dichotomy in psychology. On the one hand, there is the objective, behavioristically oriented approach, and, on the other hand, the phenomenological, existentially oriented approach. These two points of view might be thought of as mutually exclusive, but they are no more necessarily mutually exclusive than waves and particles.

It appears, in fact, that many problems with which we are acquainted, scientific, cultural, and social, have aspects to which the philosophy of complementarity may be applicable. Some time ago I saw in New York the play "J.B.," of which all of you have doubtless heard and many of you no doubt have seen. It is, you will remember, a modern dramatic pre- 
sentation of the Book of Job by Archibald MacLeish. At the climactic moment, a leading character shouts the fundamental dilemma, "If God is God, he is not good. If God is good, he is not God." After thinking about electrons, this has a strikingly familiar ring. If electrons are particles, they are not waves. If electrons are waves, they are not particles. The play as presented seems to give no very satisfactory or understandable solution to the problem. In fact, it seems to me the solution of the playwright was less clear than the solution presented by the ancient author. Without too much stretch of the imagination, one can interpret the author of Job as saying, in the language available to him, "One has no right to try to interpret God, or to interpret the universe, in terms of a limited set of ideas or an ordinary vocabulary. One must accept God, one must accept the universe." But fortunately, man need not stop there; he must continue to probe, continue to try to formulate, and thereby to understand the behavior of the universe as it is.

These various suggestions, that the epistemological lesson learned in twentieth-century physics may possibly be applicable to other fields, do not in any sense imply that the problems in other fields are solved merely by such a suggestion. The accomplishment of the physicist has been not only in the recognition of these apparent contradictions, but in the formulation of a system of quantum mechanics which can describe the results of many kinds of experiments. In this theory, the apparent contradictions in the observations are represented by the use of a mathematical device known as noncommuting operators. They are represented by rules for the statistical interpretation of wave functions. The first contribution that physics can make to other fields faced with apparent contradictions is one of encouragement. Striking success has been attained in the very simple and elementary aspects of physics. One can well take heart and believe that similar success may be attainable in more complicated problems. 
Seventeenth- and eighteenth-century physicists and mathematicians established a theory of mechanics which was rapidly adopted as a model toward which other sciences strove to develop. Twentieth-century physics also has provided an epistemological lesson which other sciences may find useful. It may well be that these contributions of science to our methods of thinking will outweigh in the long run all the material contributions which are so well known.

Last spring the director of the Oak Ridge National Laboratory in a speech to the American Rocket Association called attention to the fact that, throughout history, societies have expressed their aspirations in large-scale monumental enterprises which have taxed them to their physical and intellectual limits. The pyramids, the Sphinx, and the great temple at Karnak symbolize Egypt. The magnificent cathedrals crowning every vista of European cities reflect the culture of the Middle Ages; Versailles, the France of Louis XIV. When History looks at the twentieth century, it will see science and technology as the theme. It will find in the monuments of big science, the huge rockets, the high-energy accelerators, the great reactors, symbols of our time, just as surely as Notre Dame is a symbol of the Middle Ages.

But one may also observe that, although the pyramids and the Sphinx of Egypt survive as historical monuments, the methods of thought, the philosophies of life that grew up concurrently in Palestine and Greece are now so basic to our philosophy and our mental activity today that we rarely pause to remember their sources. Although the monuments are impressive, the pattern of thought is more fundamental. It may well be that, when the twenty-fifth century looks back on the twentieth, our tremendous engineering achievements will be superseded and our multiplicity of gadgets obsolete, but the influential and persisting elements of this century of science will be striking new ways of thinking about the physical world, in thinking about our relationship to it, and, 
most fundamentally, of thinking about ourselves and our relationship with the other human beings in it.

I have tried to present as clearly as possible one of the dilemmas we face in the slow formulation of physical law. For the formulation of physical law is a slow, hard, and continuing effort. In it there is the danger of believing the gadget is the science, rather than the philosophy which is the heart of the matter. One must not mistake the stove for the sun.

But nothing is more vital to the race of man than freedom to plumb each new idea as it comes along. The forces of reaction, as well as our own inertia, have always been opposed to such exploration.

Down at the foot of the Iberian peninsula, you will remember, are two great rocks, called the Pillars of Hercules. They are believed to have held from times long past an inscription, "ne plus ultra"-"nothing beyond." A warning to mariners, eager and young, that they would fall off the edge of the world if they ventured far beyond their familiar Mediterranean Sea.

Last night all of you heard the President of our country say that we now have a new ocean in space. So with John Glenn and his comrades we must learn to navigate it. We must not fear to do this.

For after all, are we not, on this continent, fortunate indeed that men with new ideas and new faith were brave enough five centuries ago to set sail westward into the teeth of the wind. 